\title{
Stationary Vector Autoregressive Representation of Error Correction Models
}

\author{
Yun-Yeong Kim \\ Department of International Trade, Dankook University, Yong-In, Korea \\ Email: yunyeongkim@dankook.ac.kr
}

Received December 16, 2011; revised January 20, 2012; accepted January 28, 2012

\begin{abstract}
The paper introduces a stationary vector autoregressive (VAR) representation of the error correction model (ECM). This representation explicitly regards the cointegration error a dependent variable, making the direct implementation of standard dynamic analyses using standard VAR models possible, particularly with respect to the cointegration error. Of course, an ECM does not have an explicit VAR form, and thus, it is not convenient for conducting such dynamic analyses. In this regard, we transform the original nonstationary VAR model into a VAR model with the cointegration error and stationary variables. Finally, we employ the model to dynamically analyze the real exchange rate between the US dollar and the Japanese yen.
\end{abstract}

Keywords: VAR Model; Cointegration; Error Correction Model; Stationary VAR

\section{Introduction}

The dynamic analysis of the cointegration error and stationary variables in the short run is important as the longrun equilibrium for practitioners and policy makers. Of course, such work is possible through the classical error correction model (ECM), which was popularized by Engle and Granger (1987). Furthermore, the persistence profiles of Pesaran and Shin (1996) and Hansen (2005) are also useful alternatives for this purpose.

Another possible option is to follow the vector autoregressive (VAR) approach of Sims (1980), which is now a standard method. In particular, such work may be possible if we transform the ECM into a VAR form of the cointegration error and stationary variables, which would allow the more direct exploitation of the rich tools of VAR analyses (i.e., the Granger causality test, impulse response analysis, variance decomposition, and optimal forecasting). Obviously, an ECM does not have an explicit VAR form, and thus, it is not convenient for conducting such dynamic analyses.

Then the question is whether we can transform the ECM into a finite-order VAR model of the cointegration error and stationary variables. In this regard, this paper derives a finite-order VAR model with the cointegration error that is conformable to the ECM for the short-run adjustment. In the VAR model, the cointegration error is regarded as a dependent variable, and VAR-type dynamic analyses may be conducted directly.

Finally, we employ the model to dynamically analyze the real exchange rate between the US dollar and the Japanese yen.

The rest of this paper is organized as follows. Section 2 introduces the model and assumptions. Section 3 discusses on the stationary VAR model representation of ECMs. Section 4 presents the empirical results, and Section 5 concludes.

\section{Model and Assumptions}

We consider the $\ell$-dimensionaland integrated of one $\operatorname{VAR}(p)$ process of $z_{t}$ given by

$$
z_{t}=\Pi_{1} z_{t-1}+\Pi_{2} z_{t-2}+\cdots+\Pi_{p} z_{t-p}+\varepsilon_{t}
$$

or

$$
\Delta z_{t}=\Phi z_{t-1}+\sum_{i=1}^{p-1} \Phi_{i} \Delta z_{t-i}+\varepsilon_{t},
$$

where

$$
\begin{aligned}
& \bar{\Pi}=\sum_{i=1}^{p} \Pi_{i}, \Phi=\bar{\Pi}-I_{\ell}, \\
& \Phi_{i}=-\left(\Pi_{i+1}+\Pi_{i+2}+\cdots+\Pi_{p}\right),
\end{aligned}
$$

and $\varepsilon_{t}$ is an $\ell \times 1$ vector of an independently and identically distributed disturbance term with a finite variance $\Sigma>0$, where $I_{\ell}$ denotes an $\ell$-dimensional identity matrix and $\Delta z_{t} \equiv z_{t}-z_{t-1}$.

Further we assume the cointegration of Model (1) (e.g., Johansen, 1995) as:

\section{Assumption}

We suppose $\Phi=\alpha \beta^{\prime}$, where $\alpha$ and $\beta$ are $\ell \times r$ 
matrices of the full-column rank $r$.

Note that Model (2) may be written in an ECM as

$$
\Delta z_{t}=\alpha u_{t-1}+\sum_{i=1}^{p-1} \Phi_{i} \Delta z_{t-i}+\varepsilon_{t}
$$

under Assumption 2.1, where $u_{t}=\beta^{\prime} z_{t}$.

Model (3) obviously consists of all stationary variables of $\Delta z_{t}$ and $u_{t}$ under Assumption 2.1. We are now interested in the dynamic interaction between these variables $\Delta z_{t}$ and $u_{t}$. In this regard, we may apply the results of Pesaran and Shin (1996) and Hansen (2005). However Sims' (1980) VAR approach is sometimes convenient for practical reasons. For instance, the optimal forecasting of the $k$-th-period-ahead cointegration error $u_{t+k}$ is executed mechanically in a VAR system.

Thus, we now show that Model (3) may be represented as a stationary VAR model of a part of variables $\Delta z_{t}$ and $u_{t}$ when there is an ECM.

\section{Stationary VAR Representation of ECM}

To obtain a stationary VAR representation, assume that a given cointegration vector is normalized as $\beta \equiv\left(\gamma^{\prime}, I_{r}\right)$ of the rank $r$, where $\gamma$ is $(\ell-r) \times r$. Then a confirmable non-singular square matrix can be defined as

$$
T=\left(\begin{array}{cc}
I_{\ell-r} & 0 \\
\gamma^{\prime} & I_{r}
\end{array}\right) .
$$

Noteworthy is that the above lower triangular matrix $T$ transforms the VAR variable $z_{t}=\left(x_{t}^{\prime}, y_{t}^{\prime}\right)^{\prime}$ into a variable $w_{t}$ of $x_{t}$ and cointegration error $u_{t}$.

$$
T z_{t}=\left(x_{t}^{\prime}, u_{t}^{\prime}\right)^{\prime} \equiv w_{t} .
$$

Note that $x_{t}$ is the explanatory variable of $y_{t}$ in a cointegration relation as $y_{t}=\gamma^{\prime} x_{t}+u_{t}$. We then transform the above VAR model (1) of $z_{t}$ into a VAR model of the variable $w_{t}$. In particular, we multiply the $T$ on the left of Model (1) and modify the VAR coefficients to get the following VAR model:

$$
w_{t}=\Pi_{1}^{*} w_{t-1}+\cdots+\Pi_{p}^{*} w_{t-p}+e_{t}
$$

where $\Pi_{i}^{*}=T \Pi_{i} T^{-1}$ for $i=1,2, \cdots, p$ and $e_{t}=T \varepsilon_{t}$.

Note that Model (4) is an observationally equivalent form of Model (1) or (3) if $\varepsilon_{t}$ has a Gaussian distribution. However, we cannot determine the correct model by simply analyzing observed data.

Then it is helpful to write Model (4) as

$$
w_{t}=\bar{\Pi}^{*} w_{t-1}+\sum_{i=1}^{p-1} \Phi_{i}^{*} \Delta w_{t-i}+e_{t}
$$

where $\Phi_{i}^{*} \equiv-\left(\Pi_{i+1}^{*}+\Pi_{i+2}^{*}+\cdots+\Pi_{p}^{*}\right)$ and $\bar{\Pi}^{*} \equiv \sum_{i=1}^{p} \Pi_{i}^{*}$.

Then we may simply arrange $\bar{\Pi}^{*}$ in Equation (5) as follows:

\subsection{Proposition}

Suppose that Assumption 2.1 holds. Then

$$
\bar{\Pi}^{*}=\left(\begin{array}{cc}
I_{\ell-r} & \alpha_{1} \\
0 & \beta \alpha^{\prime}+I_{r}
\end{array}\right)
$$

where $\alpha_{1}$ is $(\ell-r) \times r, \alpha_{2}$ is $r \times r$, and $\alpha \equiv\left(\alpha_{1}^{\prime}, \alpha_{2}^{\prime}\right)^{\prime}$.

Proof of Proposition 3.1. We first write from the definition

$$
\begin{aligned}
\bar{\Pi}^{*} & =T \bar{\Pi} T^{-1} \\
& =\left(\begin{array}{cc}
I_{\ell-r} & 0 \\
\gamma^{\prime} & I_{r}
\end{array}\right)\left(\begin{array}{cc}
\pi_{11} & \pi_{12} \\
\pi_{21} & \pi_{22}
\end{array}\right)\left(\begin{array}{cc}
I_{\ell-r} & 0 \\
-\gamma^{\prime} & I_{r}
\end{array}\right) \\
& =\left(\begin{array}{cc}
\pi_{11}-\pi_{12} \gamma^{\prime} & \pi_{12} \\
\gamma^{\prime} \pi_{11}+\pi_{21}-\gamma^{\prime} \pi_{12} \gamma^{\prime}-\pi_{22} \gamma^{\prime} & \gamma^{\prime} \pi_{12}+\pi_{22}
\end{array}\right)
\end{aligned}
$$

by using

$$
T^{-1}=\left(\begin{array}{cc}
I_{\ell-r} & 0 \\
-\gamma^{\prime} & I_{r}
\end{array}\right)
$$

for the first equality, where

$$
\bar{\Pi}=\left(\begin{array}{ll}
\pi_{11} & \pi_{12} \\
\pi_{21} & \pi_{22}
\end{array}\right) .
$$

We now represent the submatrices of $\bar{\Pi}$ in the third equality of (6) by using $\alpha$ and $\beta$, where $\Phi=\alpha \beta^{\prime}$ under Assumption 2.1. For this, note that

$$
\begin{aligned}
\bar{\Pi} & =\left(\begin{array}{cc}
\pi_{11} & \pi_{12} \\
\pi_{21} & \pi_{22}
\end{array}\right)=\alpha \beta^{\prime}+I_{\ell} \\
& =\left(\begin{array}{cc}
\alpha_{1} \gamma^{\prime}+I_{\ell-r} & \alpha_{1} \\
\alpha_{2} \gamma^{\prime} & \alpha_{2}+I_{r}
\end{array}\right)
\end{aligned}
$$

because the matrix $\Phi$ can be written as

$$
\Phi=\alpha \beta^{\prime}=\bar{\Pi}-I_{\ell}
$$

from the coefficient definition of (2) for the second equality.

Then the third equality in Equation (7) directly implies that

$$
\begin{aligned}
& \pi_{11}=\alpha_{1} \gamma^{\prime}+I_{\ell-r} \\
& \pi_{12}=\alpha_{1} \\
& \pi_{21}=\alpha_{2} \gamma^{\prime} \\
& \pi_{22}=\alpha_{2}+I_{r} .
\end{aligned}
$$

Consequently, if we plug the submatrices (9)-(12) into the last term in Equation (6), then we get following:

1) $\pi_{11}-\pi_{12} \gamma^{\prime}=\alpha_{1} \gamma^{\prime}+I_{\ell-r}-\alpha_{1} \gamma^{\prime}=I_{\ell-r}$

2) $\pi_{12}=\alpha_{1}$

3) $\gamma^{\prime} \pi_{11}+\pi_{21}-\gamma^{\prime} \pi_{12} \gamma^{\prime}-\pi_{22} \gamma^{\prime}$

$$
=\gamma^{\prime} \alpha_{1} \gamma^{\prime}+\gamma^{\prime}+\alpha_{2} \gamma^{\prime}-\gamma^{\prime} \alpha_{1} \gamma^{\prime}-\alpha_{2} \gamma^{\prime}-\gamma^{\prime}=0
$$

4) $\gamma^{\prime} \pi_{12}+\pi_{22}=\gamma^{\prime} \alpha_{1}+\alpha_{2}+I_{r}=\beta^{\prime} \alpha+I_{r}$.

The above results 1)-4), together with the equality in 
(6), proves the claimed result as

$$
\bar{\Pi}^{*}=\left(\begin{array}{cc}
I_{\ell-r} & \alpha_{1} \\
0 & \beta \alpha^{\prime}+I_{r}
\end{array}\right)
$$

from (6).

Note that both the dependent and explanatory variables in (5), have the nonstationary variables $x_{t}$ and $x_{t-1}$ in $w_{t}$ and $w_{t-1}$. Thus, we show how we may transform Model (5) of $w_{t}$ into a VAR model of a purely stationary variable $w_{\Delta t}=\left(\Delta x_{t}^{\prime}, u_{t}^{\prime}\right)^{\prime}$.

\subsection{Theorem}

Suppose that Assumption 2.1 holds. Then

$$
w_{\Delta t}=\Psi_{1} w_{\Delta t-1}+\Psi_{2} w_{\Delta t-2}+\cdots+\Psi_{p} w_{\Delta t-p}+e_{t}
$$

where $w_{\Delta t-i}=\left(\Delta x_{t-i}^{\prime}, u_{t-i}^{\prime}\right)^{\prime}$,

$$
\begin{aligned}
& \Phi_{i}^{*} \equiv\left(\begin{array}{cc}
\psi_{1}^{i} & \psi_{2}^{i} \\
\ell \times(\ell-r) & \ell \times r
\end{array}\right) \text { for } i=1,2, \cdots, p, \\
& \Psi_{1}=\left(\psi_{1}^{1}, \lambda+\psi_{2}^{1}\right), \quad \Psi_{i}=\left(\psi_{1}^{i}, \psi_{2}^{i}-\psi_{2}^{i-1}\right)
\end{aligned}
$$

for $i=2,3, \cdots, p-1$ and $\Psi_{p}=\left(\psi_{1}^{p-1},-\psi_{2}^{p-1}\right)$ and $\psi_{1}^{p-1}=0$.

Proof of Theorem 3.2. Using Proposition 3.1, we first rewrite Model (5) as

$$
w_{t}=\left(\begin{array}{cc}
I_{\ell-r} & \alpha_{1} \\
0 & \beta \alpha^{\prime}+I_{r}
\end{array}\right) w_{t-1}+\sum_{i=1}^{p-1} \Phi_{i}^{*} \Delta w_{t-i}+e_{t}
$$

or

$$
\left(\begin{array}{c}
\Delta x_{t} \\
u_{t}
\end{array}\right)=\left(\begin{array}{c}
\alpha_{1} \\
\beta \alpha^{\prime}+I_{r}
\end{array}\right) u_{t-1}+\sum_{i=1}^{p-1} \Phi_{i}^{*} \Delta w_{t-i}+e_{t} .
$$

The right hand side of (14) may be written as

$$
\lambda u_{t-1}+\sum_{i=1}^{p-1}\left[\psi_{1}^{i} \Delta x_{t-i}+\psi_{2}^{i}\left(u_{t-i}-u_{t-i-1}\right)\right]+e_{t}
$$

defining

$$
\lambda \equiv\left(\begin{array}{c}
\alpha_{1} \\
\beta \alpha^{\prime}+I_{r}
\end{array}\right) .
$$

Finally, the term in (15) may be written as

$$
\begin{aligned}
& \left(\lambda+\psi_{2}^{1}\right) u_{t-1}+\sum_{i=1}^{p-1} \psi_{1}^{i} \Delta x_{t-i} \\
& +\sum_{i=2}^{p-1}\left(\psi_{2}^{i}-\psi_{2}^{i-1}\right) u_{t-i}-\psi_{2}^{p-1} u_{t-p}+e_{t} \\
& =\left(\psi_{1}^{1}, \lambda+\psi_{2}^{1}\right)\left(\begin{array}{c}
\Delta x_{t-1} \\
u_{t-1}
\end{array}\right) \\
& +\sum_{i=2}^{p-1}\left(\psi_{1}^{i}, \psi_{2}^{i}-\psi_{2}^{i-1}\right)\left(\begin{array}{c}
\Delta x_{t-i} \\
u_{t-i}
\end{array}\right) \\
& +\left(0,-\psi_{2}^{p-1}\right)\left(\begin{array}{c}
\Delta x_{t-p} \\
u_{t-p}
\end{array}\right)+e_{t} .
\end{aligned}
$$

Thus, Model (14) may be rewritten as a $p$-th order
VAR model of $w_{\Delta t}$ :

$$
w_{\Delta t}=\Psi_{1} w_{\Delta t-1}+\Psi_{2} w_{\Delta t-2}+\cdots+\Psi_{p} w_{\Delta t-p}+e_{t} .
$$

Note the restrictions

$$
\psi_{1}^{p-1}=0
$$

are imposed on $\Psi_{p}$ from Theorem 3.2, and $\Delta x_{t-p}$ does not appear in Equation (13). If these restrictions (17) are not exploited and the coefficients $\psi_{1}^{p-1}$ is estimated with errors, then the efficiency of dynamic analyses by using these estimated coefficients may be reduced. For instance, if we provide forecasts using a VAR model, then forecasting mean squared error may be increased not by using restrictions (17). Campbell and Shiller (1987, Equation (5)) used the system (13) without referring to the VAR model (1) of level variable $z_{t}$, the rank deficiency of matrix $\Phi=\alpha \beta^{\prime}$ in Assumption 2.1, and the restriction of Theorem 3.2 as $\psi_{1}^{p-1}=0$ of the coefficient $\Psi_{p}$.

Further, note that the vector moving average model of $w_{\Delta t}$ is defined as $w_{\Delta t}=\Psi(L)^{-1} e_{t}$ from (13) if $\Psi(L)$ is invertible, where $L$ denotes a time-lag operator and $\Psi(L)=I-\Psi_{1} L-\cdots-\Psi_{p} L^{p}$. This is conformable to the result in Hansen (2005, Corollary1).

In the following example, we transform an ECM into a VAR model of stationary variables.

\subsection{Example}

For a VAR (2), an ECM representation can be given as

$$
\Delta z_{t}=\alpha u_{t-1}+\Phi_{1} \Delta z_{t-1}+\varepsilon_{t}
$$

under Assumption 2.1. The second-order VAR representation of $w_{\Delta t}$ is also possible as

$$
\left(\begin{array}{c}
\Delta x_{t} \\
u_{t}
\end{array}\right)=\left(\begin{array}{c}
\alpha_{1} \\
\beta \alpha^{\prime}+I_{r}
\end{array}\right) u_{t-1}+\Phi_{1}^{*}\left(\begin{array}{c}
\Delta x_{t-1} \\
\Delta u_{t-1}
\end{array}\right)+e_{t}
$$

from Model (14) by using Proposition 3.1. The righthand side of (19) may be written as

$$
\lambda u_{t-1}+\psi_{1} \Delta x_{t-1}+\psi_{2}\left(u_{t-1}-u_{t-2}\right)+e_{t}
$$

defining $\Phi_{1}^{*} \equiv\left(\psi_{1}, \psi_{2}\right) \quad$ conformably and

$$
\lambda \equiv\left(\begin{array}{c}
\alpha_{1} \\
\beta \alpha^{\prime}+I_{r}
\end{array}\right) .
$$

Finally, the term in (20) may be written as

$$
\begin{aligned}
& \left(\lambda+\psi_{2}\right) u_{t-1}+\psi_{1} \Delta x_{t-1}-\psi_{2} u_{t-2}+e_{t} \\
& =\left(\psi_{1}, \lambda+\psi_{2}\right)\left(\begin{array}{c}
\Delta x_{t-1} \\
u_{t-1}
\end{array}\right)+\left(0,-\psi_{2}\right)\left(\begin{array}{c}
\Delta x_{t-2} \\
u_{t-2}
\end{array}\right)+e_{t} .
\end{aligned}
$$

Thus, Model (19) may be rewritten as a second order VAR model of $w_{\Delta t}$ :

$$
w_{\Delta t}=\Psi_{1} w_{\Delta t-1}+\Psi_{2} w_{\Delta t-2}+e_{t}
$$

where $\Psi_{1}=\left(\psi_{1}, \lambda+\psi_{2}\right)$, and $\Psi_{2}=\left(0,-\psi_{2}\right)$ confor- 
mably, where $w_{\Delta t}=\left(\Delta x_{t}^{\prime}, u_{t}^{\prime}\right)^{\prime}$. Thus $\Delta x_{t-2}^{\prime}$ does not appear in Equation (21). Thus, the ECM (18) is written as a stationary VAR (2) model, as in (21).

Now any standard dynamic analysis is possible by using Model (13). Note that avariable $w_{\Delta t-i}=\left(\Delta x_{t-i}^{\prime}, u_{t-i}^{\prime}\right)^{\prime}$ for $i=0,1,2, \cdots, p$ is known as long as the cointegration vector $\beta$ (and thus $u_{t-i}=\beta^{\prime} z_{t-i}$ ) is known. Thus we may consistently estimate coefficients $\Psi_{i}$ in Model (13) as $\hat{\Psi}_{i}$ with variables $w_{\Delta t-i}$, using ordinary least square (OLS) method. However remind that the variable $\Delta x_{t-p}$ should be excluded from this regression.

Then we may conduct standard dynamic analyses by using the VAR model

$$
w_{\Delta t}=\hat{\Psi}_{1} w_{\Delta t-1}+\hat{\Psi}_{2} w_{\Delta t-2}+\cdots+\hat{\Psi}_{p} w_{\Delta t-p}+\hat{e}_{t}
$$

where

$$
\hat{\Psi}_{p}=\left(0,-\hat{\psi}_{2}^{p-1}\right) .
$$

Suppose cointegration vector $\beta=\left(\gamma^{\prime}, I_{r}\right)$ is not known and estimated as $\hat{\beta}=\left(\hat{\gamma}^{\prime}, I_{r}\right)$ with super-consistency as $n^{1 / 2}\left(\hat{\gamma}^{\prime}-\gamma^{\prime}\right) \rightarrow_{p} 0$ [c.f., Johansen (1995)] where $n$ is a sample number. Then $w_{\Delta t-i}$ should be replaced by
$\hat{w}_{\Delta t-i}=\left(\Delta x_{t-i}^{\prime}, \hat{u}_{t-i}^{\prime}\right)^{\prime}$ where $\hat{u}_{t-i}=\hat{\beta}^{\prime} z_{t-i}$. Thus we may consistently estimate coefficients $\Psi_{i}$ in Model (13) as $\tilde{\Psi}_{i}$ with variables $\hat{w}_{\Delta t-i}$ by using the OLS, where the variable $\Delta x_{t-p}$ should be excluded from the regression. Finally, we may conduct standard dynamic analyses by using the VAR model

$$
\hat{w}_{\Delta t}=\tilde{\Psi}_{1} \hat{w}_{\Delta t-1}+\tilde{\Psi}_{2} \hat{w}_{\Delta t-2}+\cdots+\tilde{\Psi}_{p} \hat{w}_{\Delta t-p}+\tilde{e}_{t}
$$

where

$$
\tilde{\Psi}_{p}=\left(0,-\tilde{\psi}_{2}^{p-1}\right) .
$$

In this case, we may still conduct standard dynamic analyses and draw inferences from stationary VAR analyses because $\hat{\beta}$ may be considered as known $\beta$ because of its rapid convergence. See Hamilton (1994) for a nice review on this issue.

\section{Application: An Impulse Response Analysis of the Real Exchange Rate}

The US is highly concerned about the potential exchange rate manipulation by East Asian countries for their trade

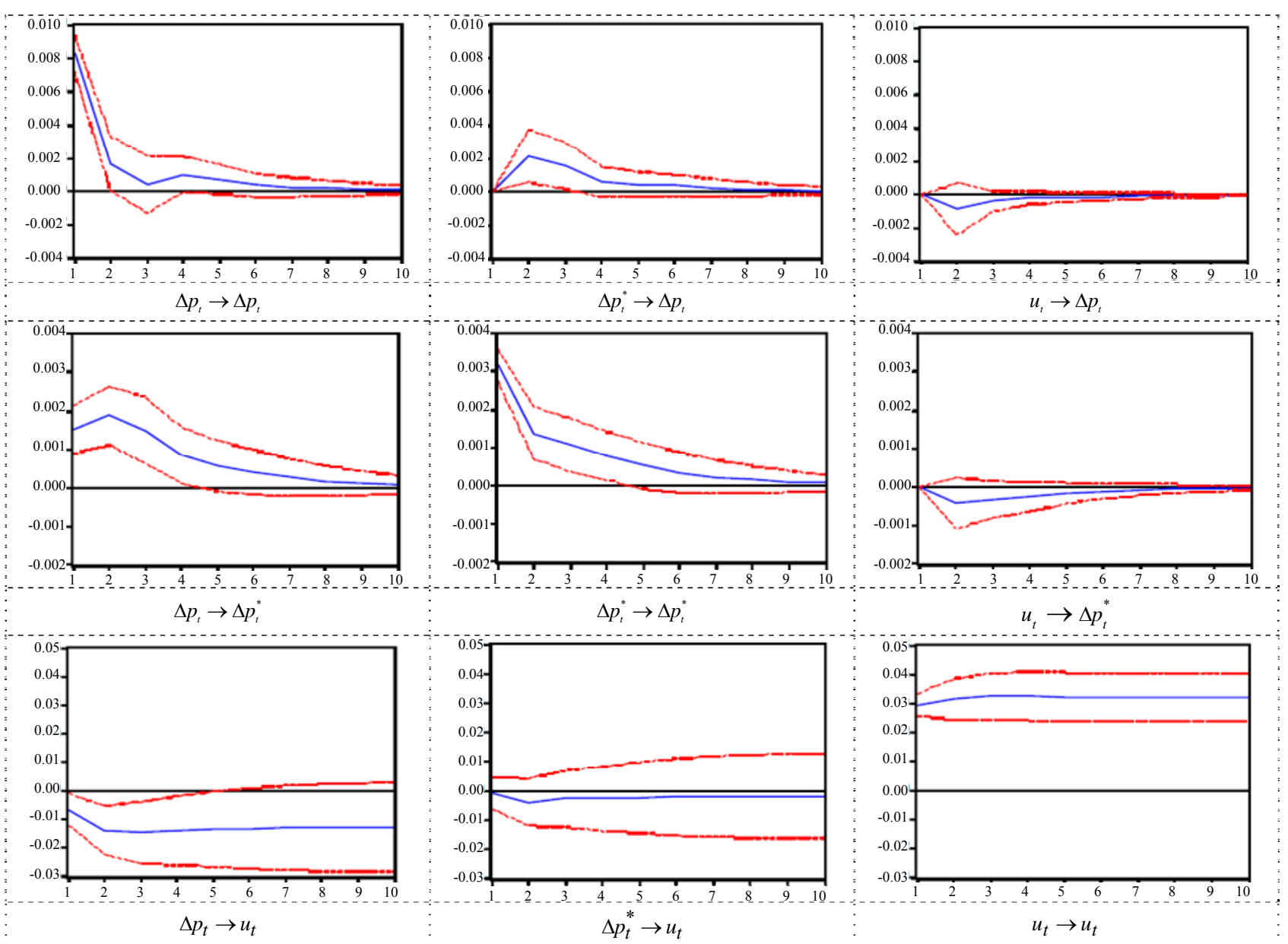

Figure 1. Impulse response analysis for USA-JAPAN PPP (response to cholesky one S.D. innovations \pm 2 S.E.). 
surplus. Krugman and Baldwin (1987) provide a classic study of this issue. See also Clarida and J. Gali (1994). The logic behind this manipulation can be explained by the law of one price (LOOP). Let $p_{t}, p_{t}^{*}$ and $f_{t}$ denote the log of the price level in the US $(P)$, the log of the Japanese price level $\left(P^{*}\right)$, and the nominal yen/dollar exchange rate $(F)$, respectively. The LOOP states that $F P^{*} / P=1$ and that the same goods should be sold at the same prices both at home and in foreign countries, where prices are converted into local prices at prevailing exchange rates. Here if the exchange rate $F$ is manipu lated as $\bar{F}$ through state intervention, then it is possible that $\bar{F} P^{*} / P>1$ in the short run, where $\bar{F} P^{*} / P$ is the relative price of the foreign good. Then the cheaper price of the home country good may induce a trade surplus.

Then the question is whether any exchange rate or price shock would have a statistically meaningful effect on the real exchange rate (a cointegration error if the real exchange rate is $I(0)$ ). To address this question, we con-

struct a VAR model of $w_{\Delta t-i}=\left(\Delta p_{t}, \Delta p_{t}^{*}, u_{t}\right)^{\prime}$ in (13), where $u_{t}=p_{t}-p_{t}^{*}-f_{t}$ and $\beta=(1,-1,-1)^{\prime}$.

Finally, we conduct an impulse response analysis on $u_{t}$ by using the Model (13). An ECM (3) does not have an explicit VAR form, and thus, it is not convenient for conducting such impulse response analysis. For this, we employ monthly data for the period from January 1998 to December 2008. We excluded the recent global financial crisis because of a possible structural break.

We conduct unit root tests for the variables $p_{t}, p_{t}^{*}$, $f_{t}$, and $u_{t}$. The results of the KPSS test do not reject the null hypothesis of stationarity at the $1 \%$ level for the cointegration error $u_{t}$ in the yen/dollar exchange rate. However, the null hypothesis of a unit root cannot not be rejected for the other variables. In a VAR model of $\left(p_{t}, p_{t}^{*}, f_{t}\right)^{\prime}$, we obtained order 2 by using the Akaike information criterion, and the results of the Johansen test reject the null hypothesis of no cointegration at the $1 \%$ level. The possible number of ordering for the identification of structural shocks is 6 for the trivariate model, and we investigate all these cases. However, changing the order has no influence on the results. Thus, we report only the result for the identification order

$\Delta p_{t} \rightarrow \Delta p_{t}^{*} \rightarrow u_{t}$. The impulses from the US price and the yen/dollar exchange rate have significant effects on the Yen/Dollar real exchange rate. See Figure 1 for the results of the impulse response analysis.

\section{Conclusion}

The ECM is clearly one of the most powerful methods in econometrics. However, it does not have an explicit VAR form with the cointegration error as a dependent variable. This paper introduces a stationary VAR representation of the ECM that explicitly regards the cointeration error as a dependent variable, making the direct implementation of standard dynamic analyses using stationary VAR models possible, particularly with respect to the cointegration error.

\section{Acknowledgements}

I am grateful to Joon Y. Park, Byeongseon Seo and the anonymous referee for their invaluable comments. All remaining errors are the author's own.

\section{REFERENCES}

[1] J. Y. Campbell and R. J. Shiller, "Cointegration and Tests of Present Value Models," Journal of Political Economy, Vol. 95, No. 5, 1987, pp. 1062-1088. doi: $10.1086 / 261502$

[2] R. Clarida and J. Gali, "Sources of Real Exchange Rate Fluctuations: How Important Are Nominal Shocks?" NBER Working Papers No. 4658, 1994.

[3] R. F. Engle and C. W. J. Granger, "Co-Integration and Error Correction: Representation, Estimation, and Testing," Econometrica, Vol. 55, No. 2, 1987, pp. 251-276. doi: $10.2307 / 1913236$

[4] J. D. Hamilton, "Time Series Analysis," Princeton University Press, Princeton, 1994.

[5] P. R. Hansen, "Granger's Representation Theorem: A Closed-Form Expression for I(1) Processes," Econometrics Journal, Vol. 8, No. 1, 2005, pp. 23-38. doi:10.1111/j.1368-423X.2005.00149.x

[6] S. Johansen, "Likelihood-Based Inference in Cointegrated Vector Autoregressive Models," Oxford University Press, New York, 1995. doi:10.1093/0198774508.001.0001

[7] P. R. Krugman and R. E. Baldwin, "The Persistence of the US Trade Deficit," Brookings Papers on Economic Activity, Economic Studies Program, The Brookings Institution, Washington, 1987, pp. 1-56.

[8] M. H. Pesaran and Y. Shin, "Cointegration and Speed of Convergence to Equilibrium," Journal of Econometrics, Vol. 71, No. 1-2, 1996, pp. 117-143. http://dx.doi.org/10.1016/0304-4076(94)01697-6.

[9] C. A. Sims, "Macroeconomics and Reality," Econometrica, Vol. 48, No. 1, 1980, pp. 1-48. doi:10.2307/1912017 